\title{
Correspondence
}

\section{Dystonic reaction after anesthesia}

To the Editor:

A healthy 24-yr-old woman had a tonsillectomy under general anesthesia with propofol, mivacurium, air, $\mathrm{O}_{2}$ isoflurane. She also received $4 \mathrm{mg}$ ondansetron and bupivacaine $0.5 \%$ with epinephrine was injected into the faucial pillars as a prophylaxis for nausea, vomiting and postoperative pain. The procedure lasted $40 \mathrm{~min}$ and the trachea was extubated when she was awake. Fifteen minutes after arrival in PACU, she started to have dys tonic movements affecting the upper limb, chest and lower limbs. The patient was awake and responsive and was very nervous. $V$ ital signs were normal, temperature $37^{\circ} \mathrm{C}$, BP $130 / 80$, heart rate 120 . She was given incremental doses of midazolam to $2 \mathrm{mg}$, but the dystonic movements persisted. The pupils were equal and reactive and patient had no pain. The differential diagnosis was adverse drug reaction, local anesthetic reaction, emergence delirium, hysterical response and post anes thetic shivering. Local anesthetic reaction is unlikely as only $5 \mathrm{ml}$ bupivacaine were given. The possibility of adverse drug reaction to ondansetron or propofol was entertained. The dystonic movements lasted three hours. Neurological consultation was requested and was thought to be a psychogenic reaction.

O ndansetron produces dystonic reactions in chronic use and propofol produces dystonic reaction, seizures and opisthotonus. ${ }^{2,3} \mathrm{H}$ ysterical reaction and delirium usually respond to sedation. Post anesthetic shivering involves the whole body and is often associated with rigidity. There is a recent report of exitatory phenomena with propofol. ${ }^{4}$

C. Ananthanarayan M D FRCPC

Kathy Blight M D

Toronto, O ntario

References

1 Wilde MI, Markham A. Ondansetron. A review of its pharmacology and preliminary clinical findings in novel applications. D rugs 1996; 52: 773-94.

2 M äkeä JA, livanainen M, Pieninkeroinen IP, Waltimo O, Lahdensuu M. Seizures associated with propofol anesthesia. E pilepsia 1993; 34: 832-5.

3 Saunders PR I, H arris M N E. O pisthotonus and other unusual neurological sequelae after outpatient anaesthesia. Anaesthesia 1990; 45: 552-7.
4 I sander G, Vinge E. Severe neuroexcitartery symptoms after anesthesia - with focus on propofol anaesthesia. Acta Anaesthesiol Scand 2000; 44: 144-9.

\section{On anesthesia services}

To the Editor:

The paper by Le $M$ ay ${ }^{1}$ reveals disquieting gaps between title and content and misconceptions about the use of words. The title appears to address issues of anesthesia service but the questionnaire includes a question concerning "adequate" relaxation. This is not a "service" (such as billing from a credit card company) rather it is a subjective evaluation by one professional about the technical performance of another although the one making the judgement is not qualified to perform that same procedure. An inept surgeon who cannot develop adequate exposure will complain about relaxation even when the patient, the anesthesiologist and the circulating nurse are all drowning in a sea of curare. Such a questionnaire should have required each surgeon to rate each anesthesiologist progressively on a case by case basis. If all the surgeons complained about the same anesthesiologist one might have proved a point. H owever, if one surgeon had complained all the time about all of the anesthesiologists then the picture would be different.

Another issue raised was that of "providing stable conditions". This is too vague to be quantified, again by somebody who is not qualified to perform that same procedure. Furthermore, at least with reference to the blood pressure, continuous automatic monitoring and recording has shown that instability of the blood pressure during anesthesia is the norm rather than the exception. The origin of the myth of the stability of the patient during anesthesia lies in the origin of the hand-made anesthesia record which in fact is a record of the anesthesiologist's perception, and what controls it, rather than a true anesthesia record.

A. Boba MD

Red H ook, N ew York, U SA

\section{Reference}

1 LeMay S, Dupuis G, H are F, Taillefer M-C, D ubéS, $H$ ardy J-F. Clinimetric scale to measure surgeons' satisfaction with anesthesia services. Can J Anesth 2000; 47: 398-405. 